\title{
Clinical features of HTLV-I associated uveitis
}

\author{
Kumiko Nakao, Norio Ohba
}

\begin{abstract}
The prevalence of human $T$ cell lymphoma virus type 1 (HTLV-I) was studied among patients with endogenous uveitis. Twelve $(15.8 \%)$ of 76 uveitis patients with known aetiology or clinical entity were seropositive, the prevalence being comparable with that in the general population of the southwestern area of Japan where HTLV-I is highly endemic. In the comparison, $32(41.0 \%)$ of 78 patients with aetiology or entity undefined uveitis were seropositive for HTLV-I, which indicated a significantly higher seroprevalence than controls matched for sex and age. The 32 cases of clinical entity undefined, HTLV-I positive uveitis were characterised by acute granulomatous or non-granulomatous uveal reactions which were accompanied by vitreous opacities and retinal vasculitis. The uveal inflammatory and retinal vascular changes responded well to topical and/or systemic corticosteroids and resolved in a few weeks in the majority of cases with favourable visual outcome. The disease affected one or both eyes, and eight cases (25\%) showed recurrence within a year. The general condition of the patients remained well otherwise during a follow up study (mean follow up time 15.4 months), except for three cases with a possible association of hyperthyroidism. These findings provide additional information favouring an association between HTLV-I and isolated uveitis, a new disease entity which should be termed HTLV-I-associated uveitis.

(Brf Ophthalmol 1993; 77: 274-279)
\end{abstract}

Human T cell lymphoma virus type 1 (HTLVI), a retrovirus distinct from human immunodeficiency virus (HIV), has been shown to cause at least two systemic diseases. One is a haematological malignancy adult $\mathrm{T}$ cell leukaemia/ lymphoma in which HTLV-I proviral DNA is integrated in the proliferating $\mathrm{T}$ lymphocytes. ${ }^{12}$ Ocular manifestations of the haematological disease include intraocular infiltration of malignant $T$ lymphocytes, intraocular opportunistic infection, such as cytomegalovirus retinitis, and adnexal lymphoma. ${ }^{3-5}$ The other disease is a chronic progressive neurological disease called HTLV-I-associated myelopathy ${ }^{6}$ or tropical spastic paraparesis.' Ocular manifestations of the neurological disease include retinal vasculitis, retinochoroidal degenerative changes, and uveitis. ${ }^{8-12}$

Our previous study showed a significantly higher prevalence of HTLV-I infection in a series of uveitis patients whose aetiology or clinical entity was undefined. It suggested an association between HTLV-I and isolated uveitis, and we proposed the name of 'HTLVI-associated uveitis (HAU)' for this clinical entity. ${ }^{13}$ However, it has remained to be established whether HTLV-1 is the causative agent of uveitis in these patients and whether such uveitis is predictive of the development of HTLV-Iassociated haematological or neurological disease. ${ }^{14}$ We have enlarged the study of uveitis patients to answer these questions and we report the results here.

\section{Materials and methods}

We studied a consecutive series of 154 patients with endogenous uveitis. There were 75 males and 79 females, and ranged in age from 12 to 87 years (mean 47.0 years, median 48.5 years). These patients were referred to the uveitis service of Kagoshima University Hospital, which is located in the southwestern area of Japan, between May 1987 and December 1991. Some patients were included who had had initial disease before 1987. The patients were followed up during the study period. Examination consisted of full physical and ophthalmic history, applanation tonometry, slit-lamp biomicroscopy with a three-mirror contact lens, and binocular ophthalmoscopy. Fluorescein fundus angiogram, electroretinogram, perimetry, and ocular ultrasonogram were obtained in selected patients. Neurological, endocrinological, and radiological examinations were performed when indicated. Laboratory studies included chest $x$ ray, assay of serum angiotensin converting enzyme, and a battery of immunoserological tests. HLA typing was performed in selected patients. Infection with HTLV-I was assessed for serum antibodies against HTLV-I using the method of particle agglutination. An agglutination reaction in patient's serum when diluted more than 16 times was regarded as seropositive. The enzyme immunoassay was also carried out on all materials to confirm the accuracy of detection of the HTLV-I antibodies.

\section{Results}

PREVALENCE OF SERUM ANTIBODIES AGAINST HTLV-I

Table 1 shows HTLV-I seroprevalence in patients with uveitis, grouped by age into specific and non-specific uveitis depending on whether the aetiology or clinical entity was defined. HTLV-I seroprevalence in the general population of the southwestern area of Japan, and published elsewhere,,$^{13}$ is also shown for reference.

Of a total of 154 patients with endogenous uveitis, 76 patients, 35 males and 41 females ranging in age from 12 to 78 years (mean $45 \cdot 0$, median 47.5 ) could be defined for aetiology or specific clinical entities including toxoplasmic retinochoroiditis, Behçet's disease, Harada's 
Table 1 Seroprevalence of antibodies to HTLV-I

\begin{tabular}{lllllllllll}
\hline \multicolumn{1}{l}{ Seroprevalence (positive cases/total cases examined) } & \\
\cline { 2 - 9 } & Age (years) & & & & \\
\cline { 2 - 9 } Disease & $10-19$ & $20-29$ & $30-39$ & $40-49$ & $50-59$ & $60-69$ & $70-79$ & $80-89$ & Total (\%) \\
\hline Specific uveitis & $0 / 4$ & $0 / 12$ & $2 / 12$ & $2 / 15$ & $4 / 16$ & $2 / 14$ & $2 / 3$ & & $12 / 76(15 \cdot 8 \%)$ \\
Non-specific uveitis & $1 / 7$ & $4 / 7$ & $8 / 14$ & $4 / 11$ & $6 / 11$ & $6 / 16$ & $1 / 9$ & $2 / 3$ & $32 / 78(41 \cdot 0 \%)^{\star}$ \\
General population $\dagger$ & & $3 / 21$ & $16 / 135$ & $110 / 614$ & $189 / 878$ & $172 / 646$ & $57 / 175$ & $5 / 15$ & $552 / 2484(22 \cdot 2 \%)$ \\
\hline
\end{tabular}

*Significantly higher seroprevalence than in general population, Mantel-Haenszel $\chi^{2}$ test $(p<0.005)$. †Data published elsewhere (ref 13$)$.

uveomeningitis, sarcoidosis, and acute anterior uveitis; $12(15 \cdot 8 \%)$ of the cases were seropositive for HTLV-I. There was no statistically significant difference in seroprevalence of HTLV-I between this group and the general population matched for sex and age.

The other group of 78 patients (age, mean 48:6; median 49 years) was undefined for aetiology or clinical entity despite full clinical and laboratory studies. Thirty two cases $(41.0 \%)$ in this group were found to be seropositive for antibodies to HTLV-I. This prevalence of seropositivity was significantly higher than that in the general population $\left(\chi^{2}=18.39, \mathrm{df}=1\right.$, $\mathrm{p}<0.005$ by Mantel-Haenszel $\chi^{2}$ test).

\section{FEATURES OF HTLV-I SEROPOSITIVE, AETIOLOGY UNDEFINED UVEITIS}

Table 2 summarises clinical data of 32 patients who had endogenous uveitis of unknown clinical entity and were seropositive for HTLV-I. There were 15 males and 17 females, and their age ranged from 17 to 87 years (mean $47 \cdot 3$, median $48 \cdot 5)$.

\section{Initial signs and symptoms}

The 32 patients presented with acute, mild to moderate visual loss frequently accompanied by visual floaters. In 10 patients, the disease was bilateral and symmetrical, and five of them had concurrent disease in both eyes. The other five had disease in the second eye within a few months after it had regressed in the first eye. The remaining 22 patients had no involvement of more than one eye during the follow up period.

Inflammatory changes were observed in the anterior and/or posterior uvea. Anterior segment reactions were present in 27 patients and vitreous opacities in 27 patients. The inflammatory signs were granulomatous in two thirds of cases and non-granulomatous in the other one third. Posterior segment changes consisted of retinal vascular changes in 13 patients, and exudative or haemorrhagic changes of the retina and choroid were observed in two patients.

Slit-lamp biomicroscopic findings were as follows. Circumcorneal injection was minimal or absent. Anterior chamber abnormalities were noted in 27 patients, consisting of $1+$ or $2+$ inflammatory cells and discrete, white keratic precipitates with minimal or absent aqueous flares. Soft, white nodules resembling the Koeppe nodule on the iris were observed in seven patients. Disorders of the angle was unremarkable; two patients had transient, mild elevation of the intraocular pressure.

Table 2 Clinical data of HTLV-I seropositive patients with aetiology undefined uveitis

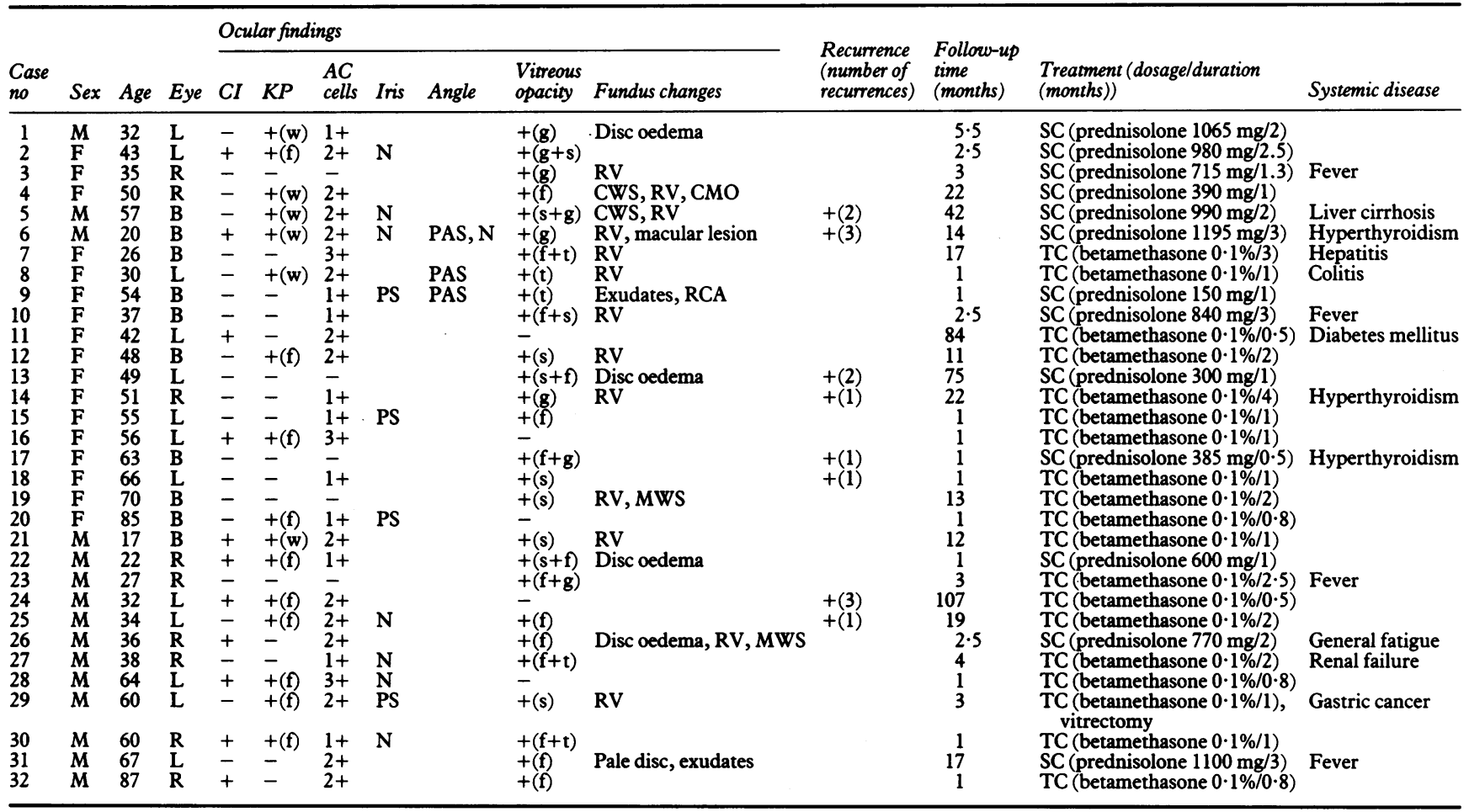

$\mathrm{CI}=$ ciliary injection; $\mathrm{KP}=$ keratic precipitate $(\mathrm{f})=$ fine,$(\mathrm{w})=$ white $\mathrm{AC}=$ anterior chamber; $\mathrm{N}=$ iris nodule; $\mathrm{PS}=$ posterior synechiae; $\mathrm{PAS}=$ peripheral anterior synechiae; vitreous opacities $(\mathrm{f})=$ fine $,(\mathrm{g})=\mathrm{granular},(\mathrm{t})=$ thread like, $(\mathrm{s})=$ snowball like; $\mathrm{RV}=$ retinal vasculitis; $\mathrm{CWS}=$ cotton wool spots; $\mathrm{CMO}=$ cystoid macular oedema; $\mathrm{RCA}=$ retinochoroidal atrophy; $\mathrm{MWS}=$ multiple white spots of retina; $\mathrm{SC}=$ systemic and topical corticosteroids, $\mathrm{TC}=$ topical corticosteroids. 
Figure 1 (case 6, Table 2) $(A, B)$ At the initial visit, white keratic precipitates and Koeppe-like nodules are observed. $(C)$

Diffuse vitreous opacities and dilated retinal veins are also present. A yellowish white, discrete round lesion is present on the superior layer of the macula. (D) Two

weeks' topical and systemic corticosteroid therapy led to a complete resolution of the anterior segment

inflammatory changes vitreous opacities, and macular lesion with normalised retinal vessels. Similar episodes with the characteristic macular lesion which resolved developed three times in a follow up of 14 months.

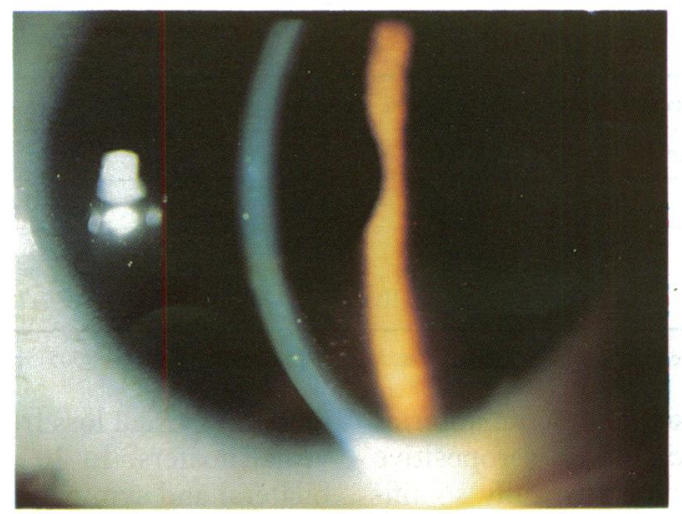

Fig $1 A$

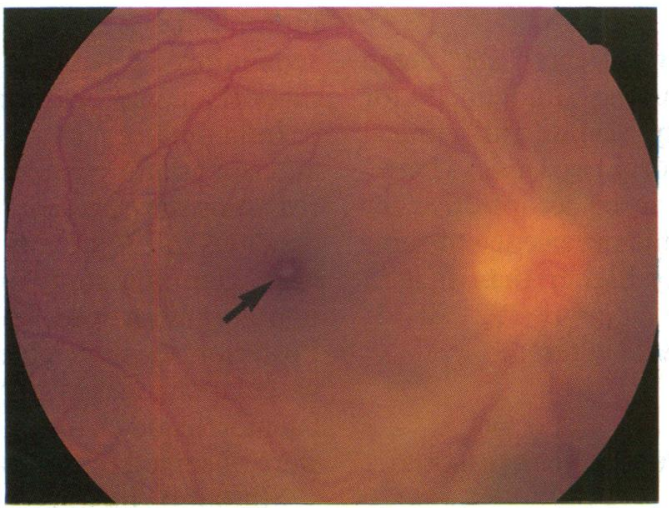

Fig 1C

Vitreous opacities of variable sizes, shapes, and densities were the most common manifestation. The lesions in the vitreous cavity consisted of aggregates of fine, white granular or threadlike opacities, snowball-like deposits, or whitishyellow membranous opacities. The density of vitreous opacities was minimal to produce visual floaters or so dense and extensive that patients complained of visual loss and the fundus was difficult to visualise.

In 13 patients ophthalmoscopy revealed mild vascular changes of the retina which consisted of punctate, white, or yellow deposits scattered on the vascular wall, frequently accompanied by similar changes in the overlying posterior hyaloid membrane. Some patients had perivascular sheathing in the posterior or peripheral

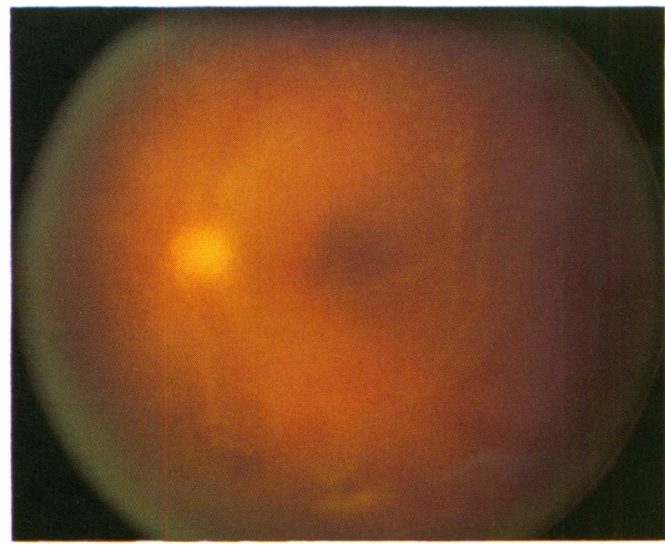

Figure 2(case 2) Soft, white, granular deposits are aggregated in the posterior vitreous cavity to reduce visibility of the fundus.

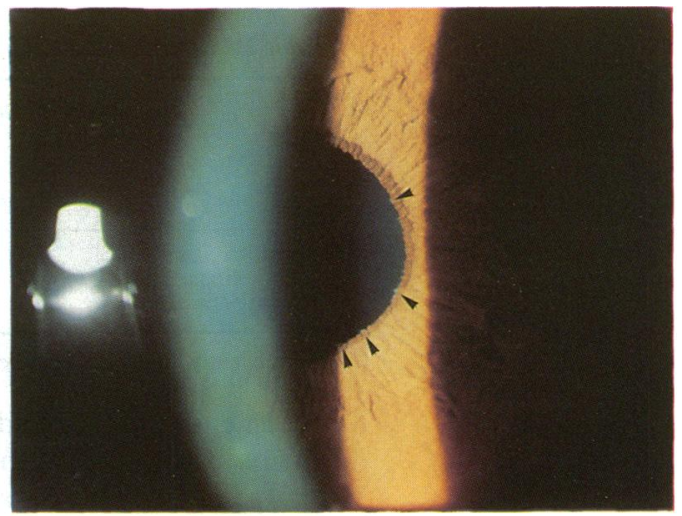

Fig $1 B$

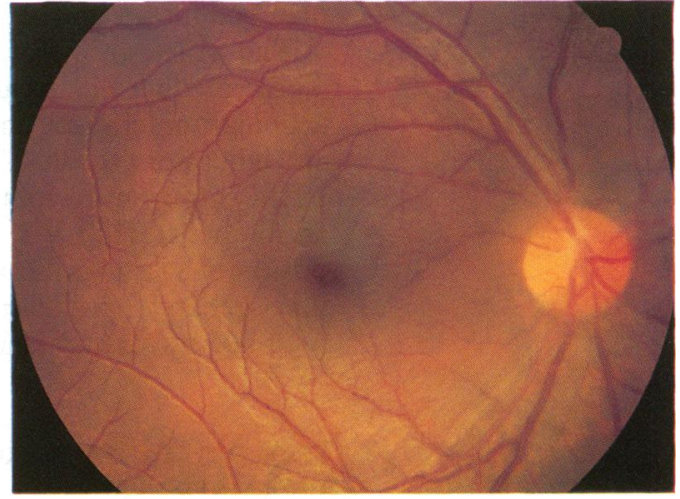

Fig $1 D$

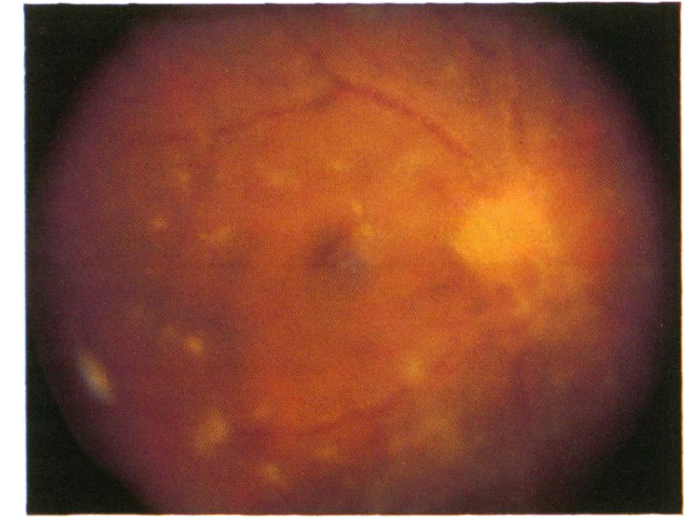

Fig $3 A$

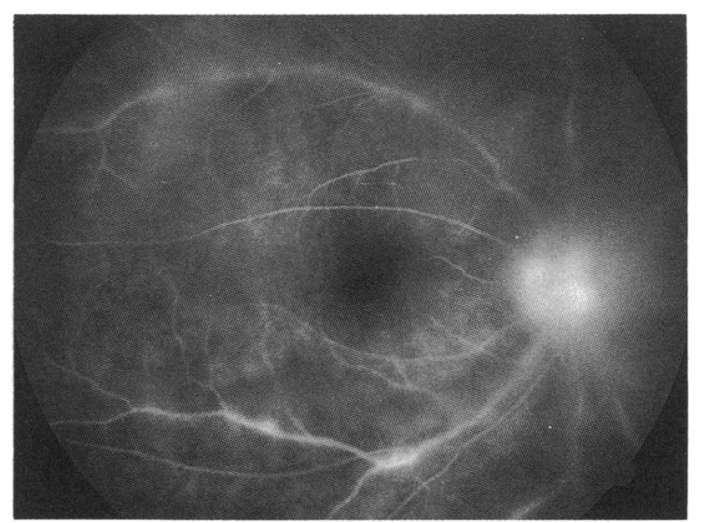

Fig $3 B$

Figure 3 (case 3) (A) Granular or snowball-like opacities are scattered in the posterior vitreous cavity to affect visibility of the fundus; retinal vessels appear distended.

(B) Fluorescein angiogram reveals considerable dye leakages from retinal veins and staining of the optic nerve head. 
Figure 4 (case 4) (A)At the initial visit, mild, diffuse vitreous haze obscures the fundus which has dilatation of retinal veins. The patient was given oral prednisolone. (B) Three weeks later, while the vitreous is cleared, multiple cotton wool spots and punctate yellow deposits on the retinal arteries have appeared. (C) Fluorescein angiogram reveals avascular areas corresponding to the cotton wool spots, and considerable leakage and staining of posterior retinal veins. Cystoid macular oedema is also obvious. (D) This photograph was taken 10 weeks after initial presentation, illustrating almost complete resolution of the retinal cotton wool spots and cystoid macular oedema, with retention of punctate deposits over the retinal arteries and some dot retinal haemorrhages. The left-hand yellowish shadow is a photographic artefact.

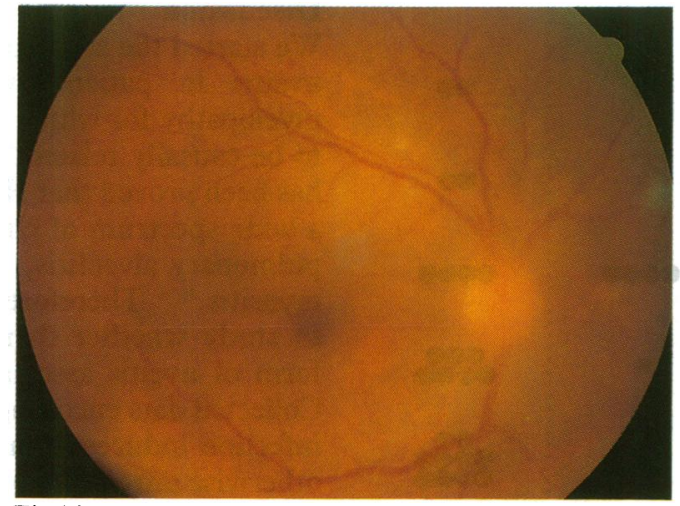

\section{Fig 4A}

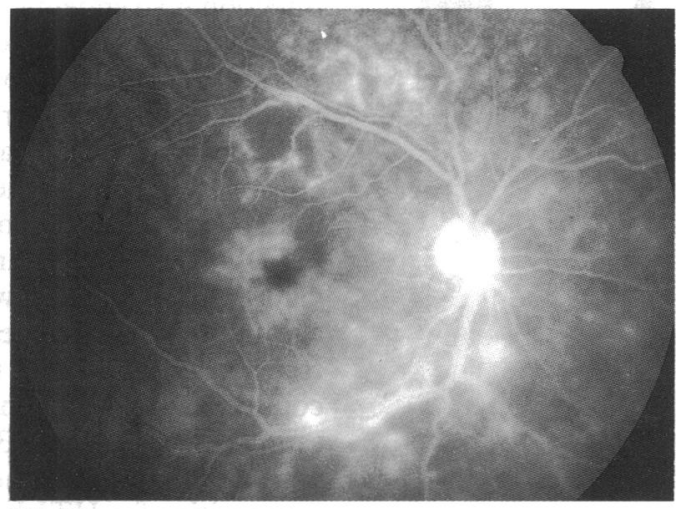

Fig 4C

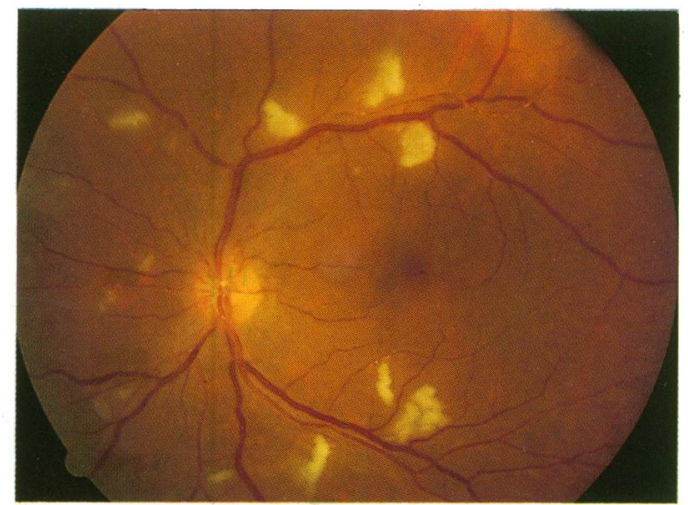

Fig 4B

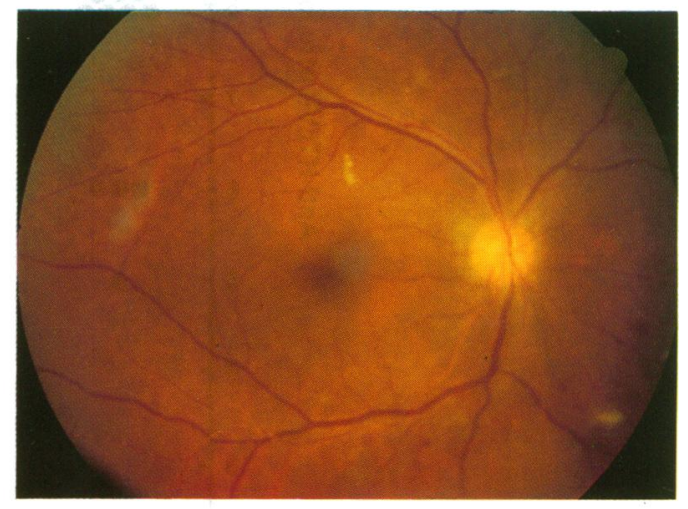

Fig $4 D$ retina, and showed dye leakage and staining on the fluorescein angiogram. Isolated or multiple cotton wool spots were also observed in some patients. The optic nerve head appeared blurred along the margin, with early dye leakage and late staining on the fluorescein angiogram in some patients. One patient (case 6 in Table 2) showed a discrete, round, white lesion located in the superficial retinal layer of the macula.

\section{Clinical course and visual outcome}

As shown in Table 2, 19 of the 32 patients were given topical corticosteroids, and 13 patients received both local and systemic corticosteroids. In the majority of cases, the uveal inflammation and retinal vasculitis subsided within a few weeks, and the visual outcome was favourable with recovery of normal visual acuity. Six patients $(18 \cdot 8 \%)$ had sequelae of iridocyclitis such as posterior synechiae or peripheral anterior synechiae. Four patients $(12 \cdot 5 \%)$ retained relatively dense, membranous opacities in the vitreous cavity which resulted in moderate visual loss. No patient had secondary glaucoma or cataract.

The follow up time ranged from 1 to 107 months (mean 15.4 months, median 3.0 months). During the follow up study, eight patients $(25 \%)$ had recurrent disease in the same eye. The recurrence occurred several months to a year after the initial presentation, with a clinical picture and course similar to the initial disease. Two patients (cases 6 and 24 in Table 2) had three episodes of recurrence during the follow up period. The clinical features are illustrated in Figures 1-4.

\section{REPORT OF CASES}

Case 3 (Table 2)

A 35-year-old woman presented with progressive floaters and blurring in the right eye. Corrected visual acuity was right eye $0 \cdot 5$, left eye $1 \cdot 5$. In the right eye anterior segments were unremarkable. There were granular opacities in the posterior vitreous and retinal veins were slightly distended. Fluorescein angiography showed considerable dye leakage from the posterior retinal veins and optic disc. With topical and oral corticosteroids, vitreous opacities resolved in 1 month, and the visual acuity returned to $1 \cdot 0$.

\section{Case 4}

A 50-year-old woman claimed acute blurring of the right vision. Corrected visual acuity was right eye 0.04 , left eye 0.9 . The right eye had white keratic precipitates, $2+$ cells in the anterior chamber and dense vitreous opacities so that the fundus was barely visible. Mild oedema of the posterior retina and punctate, white-yellow deposits over the retinal arteries were observed. Regression of the keratic precipitates and vitreous opacities in 2 weeks with topical and systemic corticosteroids was followed by manifestation of multiple cotton wool spots in the posterior fundus and cystoid macular oedema. Corticosteroid therapy was continued, and the cotton wool spots and cystoid macular oedema were almost resolved within 10 weeks with recovery of normal visual acuity, but white, punctate deposits were retained on the retinal arteries. 


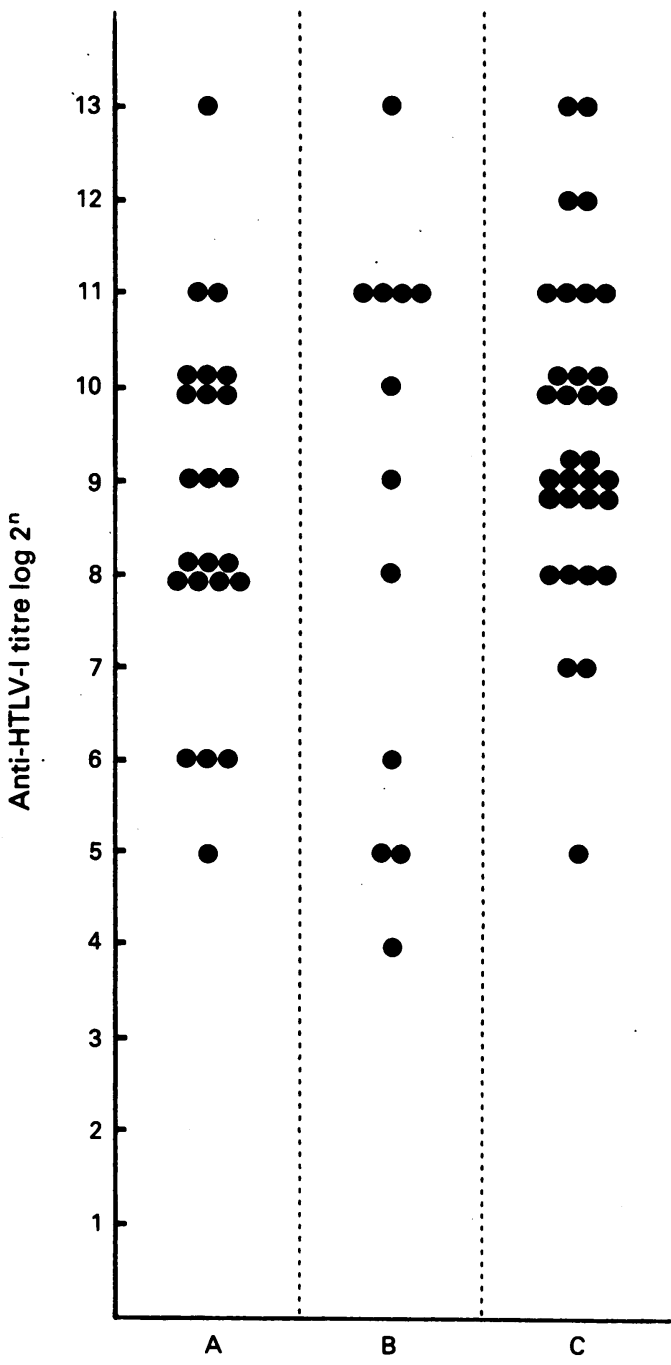

Figure 5 Distribution of serum titre of antibodies against $H T L V-I$ assessed by the particle agglutination method. $(A)$ Senile cataract patients $(n=23) ;(B)$ uveitis patients with various specific clinical entities $(n=12) ;(C)$ patients with clinical entity undefined uveitis (suspected HTLV-Iassociated uveitis) $(n=32)$.

SYSTEMIC DISEASE AND LABORATORY STUDIES

Aside from coincident systemic disorders, the majority of the 32 patients remained otherwise unremarkable, and patients did not show any sign of HTLV-I-associated haematological or neurological disorders. There were no specific findings in routine laboratory studies. HLA typing performed in 16 patients showed no specific antigen. Noticeably, three patients (cases 6, 14, 17, Table 2) had mild hyperthyroidism; one of them had mild exophthalmos, and all three patients presented with uveitis shortly after initiation of medical therapy to the systemic abnormality or after recovery of the normal thyroid function.

TITRE OF SERUM ANTIBODIES AGAINST HTLV-I The titre of serum antibodies against HTLV-I was variable among the 32 patients with clinical entity undefined uveitis, as illustrated in Figure 5. There was no difference in the distribution of serum titre among patients with suspected HTLV-I associated uveitis, asymptomatic HTLV-I carriers, and patients with uveitis of specific clinical entity.

\section{Discussion}

We started the study in 1987 when we observed uveitis in patients with HTLV-I-associated myelopathy for which the retrovirus was shown to be causally related. ${ }^{67}$ In subsequent years, it has been proved that HTLV-I infection involves a wide spectrum of clinical disorders including pulmonary alveolitis, Sjögren's syndrome, and myositis. ${ }^{15} 16$ Therefore, we thought it justified to study whether there also exists an isolated form of uveitis associated with the retrovirus. Collected data encouraged the idea that HTLV-I infection induces uveal inflammatory disease in otherwise unremarkable carriers.

In the southwestern area of Japan, HTLV-I infection is so endemic that its prevalence is $15 \%$ in adults. ${ }^{13}$ Since the majority of HTLV-I carriers remain asymptomatic throughout life, infection with the retrovirus does not directly indicate a primary cause of isolated uveitis. However, in our earlier series of 44 aetiology unknown uveitis patients, the seroprevalence of HTLV-I was $40.9 \%$ and a significantly higher value when compared with the prevalence in the age and sex matched general population. ${ }^{13}$ It has to be emphasised that the previous finding was confirmed in the present study with an increased sample size, in which $32(41.0 \%)$ of 78 patients with aetiology undefined, isolated uveitis were seropositive for HTLV-I. Hence, the retrovirus is likely to contribute to the aetiology of uveitis in some of the cases described here. Recently, another group in southwestern Japan also reported a similar uveitis to 'HTLV-I uveitis'" which probably represents the same disease we have described, and we have now started a multicentre, collaborative study to facilitate understanding of the disease. However, the descriptive term 'HTLV-I-associated uveitis' (HAU) should be adequate because the direct effect of the retrovirus on ocular tissue inflammation is unknown.

HTLV-I-associated uveitis is characterised by acute onset of inflammatory changes of the uvea, two thirds of cases presenting with granulomatous changes and the remaining cases with non-granulomatous disease, accompanied by retinal vasculitis and/or isolated or multiple cotton wool spots. The disease affects one or both eyes, apparently responds to corticosteroid therapy and regresses within a few weeks. The visual outcome is usually favourable, although moderately dense vitreous opacities are sometimes retained and cause persistent floaters and moderate visual decrease. Recurrence of the disease is not uncommon. These clinical features of HTLV-I-associated uveitis do not seem pathognomonic, and the disease should be differentiated from sarcoidosis, acute anterior uveitis, and other viral diseases which may also produce similar symptoms. It seems remarkable that uveitis with similar clinical features is found in patients with HTLV-I-associated myelopathy, ${ }^{312}$ and this provides an additional support for a possible association between HTLV-I and isolated uveitis.

Whether isolated uveitis predates HTLV-Iassociated systemic diseases remains to be elucidated. In the present follow up study (mean 15.4 months, median 3.0 months), no patient with 
isolated uveitis developed haematological or neurological disease. Aside from coincident systemic diseases in adults, it is noticeable that mild hyperthyroidism was found in three patients. A total of nine cases of association between uveitis and hyperthyroidism is described in the literature. It is interesting that one of the cases was infected with HTLV-I ${ }^{18}$ and that, although the remaining cases were not examined for HTLV-I, they were residents of the southwestern area of Japan where HTLV-I is endemic. ${ }^{1920}$

The pathomechanism of HTLV-I-associated uveitis is not clear. Our previous assessment of aqueous antibodies to HTLV-I did not provide any evidence for intraocular proliferation of the retrovirus. ${ }^{12}$ Response to corticosteroids and the recurrent nature of the disease suggest an undefined immune mechanism similar to that proposed for HTLV-I-associated myelopathy and other HTLV-I-related disease complexes $^{6}{ }^{15}{ }^{16}$; there was no specific HLA antigen in the present series of HTLV-I-associated uveitis, and the serum titre of HTLV-I-associated uveitis was comparable with that of asymptomatic carriers.

HTLV-I is widespread in the world, with endemic areas in Japan, the Caribbean, Central Africa, and South America. Many patients with HTLV-I-associated myelopathy or adult $T$ cell leukaemia/lymphoma are found not only in these endemic areas but also among immigrants in Europe and North America. This study indicates that assessment for HTLV-I infection would be worthwhile in those patients whose uveitis remains undefined for aetiology or clinical entity. Further studies are needed to support our view that HTLV-I is a causative agent of isolated uveitis. This study was supported by grants-in-aid for scientific research
(No 03771230, 03454417) from the Ministry of Education, Science and Culture of Japan.

1 Hinuma $Y$, Nagata $K$, Hanaoka $M$, Matsumoto T, Kinoshita $\mathrm{K}$, Shirakawa $\mathrm{S}$, et al. Adult T-cell leukemia: antigen in an
ATL cell line and detection of antibodies to the antigen in human sera. Proc Natl Acad Sci USA 1981; 78: 6476-80.

2 Yoshida M, Miyoshi I, Hinuma Y. Isolation and characterization of retrovirus from cell lines of human adult $\mathrm{T}$-cell leukemia and its implication in the disease. Proc Natl Acad Sci USA 1982; 79: 2031-5.

3 Ohba N, Matsumoto M, Sameshima M, Kabayama Y, Nakao $\mathrm{K}$, Unoki $\mathrm{K}$, et al. Ocular manifestations in patients with human T-lymphotropic virus type I. $\mathcal{F p n} \mathcal{F}$ Ophthalmol 1989; 33: 1-12.

4 Okubo A, Nakao K, Uemura A. A case of adult T-cell lymphoma in human $\mathrm{T}$-cell lymphoma with intraocular infiltration. $\mathcal{F}$ Eye (in Japanese) 1990; 7: 107-10.

5 Lauer SA, Fischer J, Jones J, Gartner S, Dutcher J, Hoxie JA. Orbital T-cell lymphoma in human T-cell leukemia virus type I infection. Ophthalmology 1988; 95: 110-5.

6 Osame M, Matsumoto M, Usuku K, Izumo S, Ijichi N, Amitani $\mathrm{H}$, et al. Chronic progressive myelopathy associated with elevated antibodies to human T-lymphotropic virus type 1 and adult T-cell leukemialike cells. Ann Neurol 1987; 21: 117-22.

7 Gessain A, Barin F, Vernant JC, Gout O, Maurs L, Calender A, et al. Antibodies to human T-lymphotropic virus type-1 in patients with tropical spastic paraparesis. Lancet 1985; ii: in patien. 10 .

8 Nakao K, Uemura A, Hirashima S, Unoki K. Cotton-wool spots on the retina in patients infected with human $\mathrm{T}$ lymphotropic virus type 1 (HTLV-I). Folia Ophthalmol fpn 1989; 40: 2051-7.

9 Sasaki K, Morooka A, Inomata H, Akamine T, Osame $M$. Retinal vasculitis in human $\mathrm{T}$-lymphotropic virus type 1 associated myelopathy. Br F Ophthalmol 1989; 73: 812-5.

10 Hayasaka S, Takatori Y, Noda S, Setogawa T, Hayashi H. Retinal vasculitis in a mother and her son with human T-lymphotropic virus type 1 associated myelopathy. Br\} Ophthalmol 1991; 75: 566-7.

11 Nakao K, Ohba N, Isashiki M, Isashiki Y, Unoki K, Osame M. Pigmentary retinal degeneration in patients with HTLVI-associated myelopathy. Fpn f Ophthalmol 1989; 33: 38391 .

12 Nakao K, Ohba N, Matsumoto M. Noninfectious anterior uveitis in patients with human T-lymphotropic virus type 1 . uveitis in patients with human T-lym

13 Nakao K, Matsumoto M, Ohba N. Seroprevalence of antibodies to HTLV-I in patients with ocular disorders. $\mathrm{Br} F$ Ophthalmol 1991; 75: 76-8.

14 Spalton DJ, Nicholson F. HTLV-I infection in human disease. Br f Ophthalmol 1991; 75: 174-5.

15 Vernant JC, Buisson G, Magdeleine J, DeThore J, Jouannelle $A$, Neisson-Vernant $C$, et al. T-lymphocytic alveolitis, tropical spastic paraparesis, and Sjögren's syndrome. Lancet 1988; i: 177.

16 Morgan OS, Rodgers-Johnson P, Mora C, Char G. HTLV-I and polymyositis in Jamaica. Lancet 1989; ii: 1184-7.

17 Mochizuki M, Watanabe T, Yamaguchi K, Takatsuki K, Yoshimura K, Shirao M, et al. HTLV-I uveitis: a distinct clinical entity caused by HTLV-I. F p $\mathcal{F}$ Cancer Res 1992; 83: 236-9.

18 Yamamoto S, Kakisu Y. Severe uveitis developed in a case of dysthyroid ophthalmopathy [in Japanese]. Ganka Rinsho dysthyroid ophthalmopathy [in Japanese].

19 Mizoguchi T, Sato T, Dake Y, Amemiya T. Hyperthyroidism and uveitis [in Japanese]. Rinshoganka (fpn $\mathcal{J}$ Clin Ophthalmol) 1990; 44: 908-9.

20 Shimada E, Yamanaka H, Hirayama Y, Mizoguchi T, Amemiya $T$. Uveitis in three patients with hyperthyroidism [in Japanese]. Folia Ophthalmol f pn 1991; 42: 1755-60. 